\title{
No need for broad-spectrum empirical antibiotic coverage after surgical drainage of orthopaedic implant infections
}

\author{
Maximilian Schindler • Axel Gamulin • Wilson Belaieff • \\ Maruschka Francescato • Alexis Bonvin • Véronique Graf • \\ Pierre Hoffmeyer • Blaise Wyssa • Ilker Uçkay
}

Received: 20 March 2013 /Accepted: 28 April 2013 /Published online: 7 June 2013

(C) Springer-Verlag Berlin Heidelberg 2013

\begin{abstract}
Purpose Empirical broad-spectrum antibiotic treatment for orthopaedic implant infections after surgical lavage is common practice while awaiting microbiological results, but lacks evidence.

Methods This was a single-centre cohort study from 1996 to 2010 with a follow-up of two years.

Results We retrieved 342 implant infections and followed them up for a median of 3.5 years (61 recurred, $18 \%$ ). Infected implants were arthroplasties $(n=186)$, different plates, nails or other osteosyntheses. The main pathogens were $S$. aureus (163, 49 methicillin-resistant) and coagulasenegative staphylococci (60, 45 methicillin-resistant). Median duration of empirical antibiotic coverage after surgical drainage was three days before switching to targeted therapy. Vancomycin was the most frequent initial empirical agent (147), followed by intravenous co-amoxiclav (44). Most empirical antibiotic regimens $(269,79 \%)$ proved sensitive to the causative pathogen, but were too broad in 111 episodes (32\%). Cephalosporins and penicillins were used only in 44 and ten cases, respectively, although they would have covered
\end{abstract}

M. Schindler · A. Gamulin · W. Belaieff · M. Francescato •

A. Bonvin · P. Hoffmeyer • B. Wyssa $\cdot$ I. Uçkay $(\bowtie)$

Orthopedic Surgery Service, Geneva University Hospitals \&

Faculty of Medicine, University of Geneva, 4, Rue Gabrielle

Perret-Gentil, 1211 Geneva 14, Switzerland

e-mail: ilker.uckay@hcuge.ch

M. Schindler $\cdot$ V. Graf

Service of Rehabilitation, Geneva University Hospitals \& Faculty

of Medicine, University of Geneva, Geneva, Switzerland

I. Uçkay

Service of Infectious Diseases, Geneva University Hospitals \&

Faculty of Medicine, University of Geneva, Geneva, Switzerland
$59 \%$ of causative pathogens identified later. Multivariate Cox regression analysis showed that neither susceptible antibiotic coverage (compared to non-susceptible; hazard ratio 0.7, $95 \%$ confidence interval $0.4-1.2)$ nor broad-spectrum use (hazard ratio 1.1, 0.8-1.5) changed remission rates. Conclusions Provided that surgical drainage is performed, broad-spectrum antibiotic coverage does not enhance remission of orthopaedic implant infections during the first three days. If empirical agents are prescribed from the first day of infection, narrow-spectrum penicillins or cephalosporins can be considered to avoid unnecessary broad-spectrum antibiotic use.

\section{Introduction}

During debridement and/or removal of infected implants, orthopaedic surgeons perform intraoperative microbiological sampling and start empirical antibiotic therapy. Because the pathogen is often unknown at debridement and due to doubts of polymicrobial and resistant nosocomial infections, this initial coverage is usually too large in the antibiotic spectrum. Sometimes, also the local epidemiology with potentially resistant germs determines the choice of empirical antimicrobial therapy and not only the desire to cover all pathogens. However, existing recommendations and guidelines address choices and durations of targeted antibiotic therapy [1,2], but do not propose initial empirical coverage, which is left to the discretion of the treating surgeon [3]. Many colleagues think that it would be beneficial if empirical antibiotic administration could cover all possible pathogens for arthroplasty [2] and other orthopaedic infections [4], with usual recurrence rates oscillating between 15 and $28 \%$ [2, 5, 6], and especially in cases of implant retention. Experimentally speaking, biofilms are established within a few hours or days [7], and infections 
with resistant micro-organisms may harbour more treatment failures than infections due to susceptible bacteria [5]. Therefore it is not surprising that many authors routinely administer vancomycin, alone [3] or in combination with meropenem $[2,3]$, or aminoglycosides [4] or other antipseudomonal drugs [4], until the pathogen is identified.

However, antibiotic excesses, even of short duration, contribute to the spread of multi-resistant pathogens $[8,9]$ and might harm the patient, e.g. antibiotic-related diarrhoea in its multiple forms [2]. The prolongation of antibiotic administration beyond 24 hours after surgery favours the acquisition of antibiotic resistance, particularly among Gram-negative pathogens [9], which may become responsible for epidemics in septic orthopaedic wards.

In this study, we postulate that surgical drainage is the most important therapeutic action, while the antibiotic coverage during the initial days does not influence outcome.

\section{Methods}

Geneva University Hospitals serve as a 2,000-bed tertiary teaching hospital and the only public health institution in the canton (county). The Orthopedic Service has 132 beds and has conducted an arthroplasty cohort since 1996, together with several prospective databases regarding orthopaedic infections. Patients are followed up after hospital discharge until remission of infection. Arthroplasty patients are followed up at one year and five years, even in the absence of problems.

\section{Data collection and definitions}

MS and IU retrospectively collected 61 variables of orthopaedic implant infections in adults from January 1996 to December 2010. The last day of active follow-up was 31 December 2012. We excluded recurrent episodes, paediatric cases, patients in the intensive care units and those that had been treated by antibiotics before admission [10]. The diagnosis of implant infection was based upon the presence of intraoperative pus together with clinical signs of infection (new onset of pain, fever, sinus tract, discharge) and/or radiographic signs of implant loosening. Species identification required the same pathogen to be present in at least two intraoperative samples [11]. This study is about the active spectrum of prescribed antibiotic agents. For our study, we defined what we considered as adequate and what spectrum was too broad for the individual pathogen. In the literature, there is only flawed consensus on what is regarded as "too broad" and what is not, depending on the local medical culture. Empirical antibiotic treatment was considered too broad when the applied agent exceeded the minimum applicable spectrum defined as the use of penicillin, amoxicillin, cefuroxime, cefamandole, clindamycin, co-trimoxazole, flucloxacillin and depending on the pathogen. These agents cover most of the Gram-positive pathogens, the hallmark of orthopaedic infections. By study definition, broad-spectrum antibiotic therapy covered more than necessary. Consequently, co-amoxiclav, ceftriaxone, ceftazidime, cefepime, piperacillin/tazobactam, carbapenems, quinolones, daptomycin, linezolid, tigecycline, systemic or local aminoglycosides or glycopeptides were considered too broad if the pathogen was susceptible to narrowspectrum coverage. In contrast, it was considered adequate if the bacteria were resistant, leaving no choice for a narrower spectrum.

\section{Statistical analyses}

Group comparisons were performed using the Pearson's $\chi^{2}$ or the Wilcoxon rank sum test, as appropriate. A Cox regression analysis determined association with outcome remission. Only first episodes of implant infections were included. Independent variables with a $p$ value $\leq 0.2$ in univariate analysis were introduced stepwise in the multivariate analysis [12]. Key variables regarding antibiotic coverage were introduced into the final model independently of univariate results. All variables were checked for confounding and interaction: the latter by interaction terms and Mantel-Haenszel estimates. A $p$ value $\leq 0.05$ (two-tailed) was significant. Stata ${ }^{\mathrm{TM}}$ software (9.0, StataCorp, College Station, TX, USA) was used.

\section{Results}

Patients and infections

A total of 342 infections in 342 patients (133 women) met the study criteria and were actively followed up for a median of 3.5 years (range, two to 15 years). The median age was 67 years (range 18-94 years) and 109 patients (32\%) were immunocompromised due to diabetes mellitus $(n=41)$, active cancer (25), severe alcoholism (16), steroid medication (13), Child's class C cirrhosis (4), uncontrolled human immunodeficiency virus (HIV) disease (2), splenectomy (1), dialysis (1) and and organ transplantation (1). Six patients suffered from combinations.

The infected implants were 113 hip arthroplasties, 68 knee arthroplasties, two ankle prostheses, one shoulder prosthesis, one elbow prosthesis and one unicompartmental knee implant. The remaining 156 (46\%) infected implants were dynamic hip screws, plates, screws, nails and external fixations in various bones. Pathogens varied considerably. We found 36 different monomicrobial and 18 polymicrobial episodes [11]. Sonication was not performed. The main germ was methicillin-sensitive Staphylococcus aureus $(114,33 \%)$, followed by coagulase-negative staphylococci $(60,17 \%)$ and methicillin-resistant $S$. aureus (MRSA; 49, 
$14 \%$ ). Enterococci were encountered in seven infections. Gram-negative infections were a minority $(51,15 \%)$, including 18 episodes due to Pseudomonas spp., 11 other nonfermenting rods and two extended-spectrum beta-lactamase producers. Strict anaerobes were detected in five cases, three of which were co-pathogens. Overall, 75 episodes were bacteraemic without haemodynamically compromising sepsis.

\section{Antibiotic treatment}

The median duration of empirical treatment was three days (range 0-18 days). Overall, single-agent use (246) was more frequent than combination therapy (67). The most frequent agent used was vancomycin $(n=147)$, solely (89) or in combination. Other empirical antibiotics were co-amoxiclav (44 episodes), quinolones (23), flucloxacillin (21), aminoglycosides (18), carbapenems (15), cefepime (15), ceftriaxone (10), clindamycin (13) and piperacillin/tazobactam (2). Co-trimoxazole, daptomycin, fusidic acid, metronidazole, teicoplanin and ceftazidime were mostly co-administered with other antimicrobials in less than three cases each.

The majority of antibiotic regimens (269/342, $79 \%$ ) proved to be sensitive to the later identified pathogen, but was too broad in 111 cases (32\%). Of note, 29 patients (8 \%) did not receive any empirical treatment, because infection was diagnosed only when intraoperative specimens became positive. Anaerobic coverage with mostly amoxicillin/clavulanate or clindamycin was performed in 130 episodes (38\%), although only three pathogens were real strict anaerobes, while we could not elucidate why exactly the treating physicians chose agents with concomitant anaerobic coverage. Penicillin (including amoxicillin) and first- and second-generation cephalosporins were used in ten and 44 cases, respectively, although they would have covered $59 \%$ of all later identified pathogens. Once the pathogen was identified, the overall median duration of targeted antimicrobial therapy was 56 days (range 14-294 days), of which a median of 14 days intravenously (range 0-100 days).

\section{Surgical treatment}

Surgery was part of treatment for every episode with a mean and median number of two interventions (range 0-17). The number of surgical interventions between episodes with broad-spectrum compared to narrow-spectrum coverage was similar (median two vs two interventions, $p=0.77$ ). In 86 episodes $(25 \%)$, the infected implant was retained. Among the 186 arthroplasty infections, 86 (46\%) were treated with debridement and retention alone, 7 (4\%) were removed without further implantation and 91 (49\%) episodes underwent a two-stage exchange with a median interval of nine weeks between the two stages. Only two onestage arthroplasty exchanges were performed.
Outcomes and multivariate analysis

Among all 342 episodes of implant infections, 61 (18\%) clinically recurred after a median of 5.5 months with identical pathogens. The patient populations with and without recurrences were similar with the exception of arthroplasty retention and immunosuppression (Table 1). Because of a substantial variety of pathogens, antibiotics and implants, we performed a Cox regression analysis to adjust for case mix (Table 2). In multivariate analysis, no variable was associated with recurrence. Adequate choice of empirical drugs [hazard ratio (HR) 0.7, $95 \%$ confidence interval (CI) 0.4-1.2] [reciprocal: wrong antibiotic coverage (HR 1.1, 0.8-1.5)], broad-spectrum coverage (HR 1.1, 0.8-1.5) or duration of vancomycin use (HR 1.0, 1.0-1.0) failed to enhance remission. Of note, the receiver-operating characteristic curve value was 0.80 , highlighting an acceptable accuracy of our final model.

\section{Discussion}

Among 342 adult patients with implant infections, one fifth underwent an empirical antibiotic treatment post-drainage that did not cover the later identified pathogen, and one third witnessed excessive coverage in our retrospective evaluation of daily clinical practice. However, broad-spectrum antibiotic coverage failed to enhance remission or to reduce the number of surgical interventions. Equally, empirical use of vancomycin showed no protective effect, although in our institution the MRSA endemicity among clinical $S$. aureus isolates is around $28 \%$ [13] and $75 \%$ of clinical coagulasenegative staphylococci are methicillin-resistant [14]. At the same time, we failed to prove any inconvenience for the episodes where empirical therapy was wrongly chosen during the first three days.

Many patients witnessed treatment against anaerobes, although finally there were only five anaerobic (co)-pathogens detected. Regarding this anaerobic coverage, we could not detect why the treating surgeons and physicians chose antimicrobial agents active against anaerobic pathogens. Retrospectively, narrow-spectrum penicillins or secondgeneration cephalosporins alone would have correctly covered two thirds of the pathogens.

We would discourage the use of routine empirical vancomycin coverage. Our opinion is in line with experts denying the existence of a threshold of resistant staphylococcal surgical site infections $[13,14]$, above which a switch to glycopeptides would be necessary in terms of prevention and empirical treatment $[8,15]$. Authors from the Mayo Clinic successfully treated culture-negative prosthetic joint infections throughout the course with cefazolin alone, with no excess of treatment failure (HR $0.7,95 \%$ CI $0.2-3.0$ ) 
Table 1 Comparison of episodes with and without recurrence of implant infections

\begin{tabular}{|c|c|c|c|c|}
\hline & $\begin{array}{l}\text { Numbers, } \\
n=342\end{array}$ & $\begin{array}{l}\text { Recurrence, } \\
n=61\end{array}$ & $p^{\mathrm{a}}$ & $\begin{array}{l}\text { Remission, } \\
n=281\end{array}$ \\
\hline Female gender & 133 & $19(31 \%)$ & & $114(41 \%)$ \\
\hline Median age & & 64 years & & 67 years \\
\hline Immunosuppression $^{\mathrm{b}}$ & 109 & $26(43 \%)$ & 0.047 & $83(30 \%)$ \\
\hline Arthroplasty infection & 186 & $31(51 \%)$ & & $155(55 \%)$ \\
\hline Retained arthroplasties & 86 & $20(33 \%)$ & 0.042 & $66(24 \%)$ \\
\hline Two-stage exchanges & 91 & $11(18 \%)$ & & $80(28 \%)$ \\
\hline Bacteraemia & 75 & $14(23 \%)$ & & $61(22 \%)$ \\
\hline MRSA infection ${ }^{\mathrm{c}}$ & 49 & $11(18 \%)$ & & $38(14 \%)$ \\
\hline Gram-negative infection & 51 & $10(16 \%)$ & & $41(15 \%)$ \\
\hline Empirical vancomycin use & 147 & $28(46 \%)$ & & $119(42 \%)$ \\
\hline Correct empirical antibiotic coverage & 269 & $44(72 \%)$ & & $225(80 \%)$ \\
\hline Too broad spectrum use & 111 & $21(34 \%)$ & & $90(32 \%)$ \\
\hline Median duration of empirical antibiotics & & 3 days & & 3 days \\
\hline Median duration of total antibiotic use & & 60 days & & 56 days \\
\hline Median duration of IV antibiotics & & 14 days & & 14 days \\
\hline Median no. of surgical interventions & & 2 interventions & & 2 interventions \\
\hline
\end{tabular}

${ }^{\text {a }}$ Pearson's $\chi^{2}$ test. Only significant values $(p$ value $<0.05)$ are displayed

${ }^{\mathrm{b}}$ diabetes, cancer, alcoholism, steroid medication, cirrhosis, HIV, splenectomy, dialysis

${ }^{\mathrm{c}}$ Methicillin-resistant Staphylococcus aureus

Table 2 Cox regression analysis with outcome remission of orthopaedic implant infections

n.a. omitted from final model because of interaction or limited number of variables

${ }^{a}$ Diabetes, cancer, alcoholism, steroid medication, cirrhosis, HIV, splenectomy, dialysis

${ }^{\mathrm{b}}$ Methicillin-resistant

Staphylococcus aureus

\begin{tabular}{|c|c|c|}
\hline Variable & $\begin{array}{l}\text { Univariate analysis } \\
\mathrm{HR}, 95 \% \mathrm{CI}\end{array}$ & $\begin{array}{l}\text { Multivariate analysis } \\
\mathrm{HR}, 95 \% \mathrm{CI}\end{array}$ \\
\hline Female gender & $1.0,0.8-1.3$ & n.a. \\
\hline Age & $1.0,1.0-1.0$ & n.a. \\
\hline Age 65 years compared to $<65$ years & $1.1,0.9-1.4$ & n.a. \\
\hline Arthroplasty infection & $1.0,0.8-1.3$ & n.a. \\
\hline Arthroplasty retention & $1.2,0.8-1.7$ & n.a. \\
\hline Two-stage exchanges & $0.8,0.5-1.04$ & n.a. \\
\hline All implant retentions & $1.2,0.9-1.6$ & $1.4,0.8-2.3$ \\
\hline Immunosuppression $^{\mathrm{a}}$ & $1.1,0.8-1.4$ & $1.4,0.9-2.2$ \\
\hline MRSA infection ${ }^{\mathrm{b}}$ & $1.1,0.8-1.5$ & n.a. \\
\hline Gram-negative infection & $1.3,0.9-1.8$ & n.a. \\
\hline Due to Pseudomonas spp. & $1.3,0.8-2.2$ & n.a. \\
\hline Polymicrobial infection & $1.2,0.7-2.3$ & n.a. \\
\hline No. of surgical interventions & $1.0,0.9-1.1$ & $1.0,0.9-1.1$ \\
\hline \multicolumn{3}{|l|}{ Antibiotic-related parameters } \\
\hline Empirical vancomycin use & $1.2,0.9-1.5$ & n.a. \\
\hline Duration of vancomycin use & $1.0,1.0-1.0$ & $1.0,1.0-1.0$ \\
\hline Correct empirical coverage & $0.8,0.6-1.1$ & $0.7,0.4-1.2$ \\
\hline (Wrong empirical coverage) & $1.2,0.9-1.6$ & $1.1,0.8-1.5)$ \\
\hline Too broad spectrum use & $1.2,0.9-1.5$ & $1.1,0.8-1.5$ \\
\hline Duration of empirical antibiotics & $1.0,0.9-1.1$ & $1.0,0.9-1.1$ \\
\hline Duration of total antibiotic use & $1.0,1.0-1.0$ & n.a. \\
\hline $6-12$ weeks compared to $<6$ weeks & $1.1,0.9-1.5$ & $1.1,0.7-1.7$ \\
\hline$>12$ weeks compared to $<6$ weeks & $1.1,0.8-1.5$ & $1.1,0.7-2.0$ \\
\hline
\end{tabular}


[17]. Despite widespread practice and recommendations, few studies scientifically advocate universal empirical vancomycin use after lavage. However, they may stem from institutions where the proportion of MRSA infections was $64 \%$, compared to $14 \%$ in our study [3].

One theoretical concern of our study relates to retained implants, especially retained arthroplasties. Biofilm formation starts within a very short period of time [7]. If an implant is removed (and therefore the biofilm is removed with the implant), it is conceivable that potentially incorrect antibiotic therapy has no influence on the outcome. In contrast, it might be difficult to understand why an inadequate empirical antibiotic therapy has no influence on treatment failure when the attached implant has not been removed, as we show in this study.

For us, this discrepancy between theoretical concern and practice is not surprising for various reasons. First, empirical antibiotic coverage only lasted for the first three days (e.g. $7 \%$ of the total duration), and not the rest of the antibiotic prescription $(93 \%)$. Second, even if the prosthesis was retained, the patient underwent at least one thorough debridement, which is more important in mechanically removing the biofilm than the chemical effect of antibiotic drugs, which combat bacteria but not the matrix of the biofilm per se. Finally, biofilm formation within hours is supported by in vitro data, but no one knows the time delay above which this biofilm becomes clinically decisive. In the literature, some groups of expert researchers advocate that, in vitro, biofilms are established rapidly, but in vivo, they still allow clinicians to retain the prosthesis up to three weeks after the onset of infection [1, 17].

In conclusion, we think the most important part in the treatment of orthopaedic implant infections is surgical drainage, long before antibiotics show benefit. The effect of this initial lavage is substantial, reducing microbial inoculum and removing pus, independently of whether or not the implant has been retained. Penicillins or cephalosporins might be sufficient to bridge the short time until availability of microbiological results, even if this narrow spectrum does not cover the pathogen. We therefore discourage the use of routine empirical vancomycin or other broad-spectrum coverage, unless the local epidemiology is unusual or there is currently an outbreak situation due to resistant pathogens. If future prospective trials confirm our findings, there is space for antibiotic stewardship, cost savings and reduction of potential spread of antibiotic resistance in orthopaedic wards [9].

Our study has biases: (1) It is a single-centre retrospective study, limiting the general application of the results. Although including 342 episodes, this number might be too small to detect differences in the outcome. Concerning substrata of implant infections, only 86 implants were retained, and only two witnessed a one-stage arthroplasty exchange, further limiting the generalisability of our data. (2) Our patients had no haemodynamic compromises. For patients in intensive care, adequate initial empirical antibiotic coverage is crucial and may be lifesaving. (3) Patients may have been followed elsewhere. However, because Geneva University Hospitals are the only public hospital in the area, and most arthroplasty patients participate in our register, we estimate this bias as minimal. (4) With only 29 episodes without any empirical antibiotics at all, we cannot pronounce on the question of whether antibiotic coverage per se would be necessary. This question needs a much larger study population. (5) The duration of empirical therapy peaked around three days, which should not be confounded with culture-negative therapy throughout the course. The literature is divided on whether a culture-negative therapy would [6] or would not be $[10,16]$ associated with higher failure risk.

Acknowledgments We thank all colleagues of the Orthopedic Service of Geneva University Hospitals. Ilker Uçkay had full access to all of the data in the study and takes responsibility for the integrity of the data and the accuracy of the data analysis.

Conflict of interest The authors declare that they have no conflict of interest.

\section{References}

1. Osmon DR, Berbari EF, Berendt AR, Lew D, Zimmerli W, Steckelberg JM, Rao N, Hanssen A, Wilson WR (2013) Diagnosis and management of prosthetic joint infection: clinical practice guidelines by the Infectious Diseases Society of America. Clin Infect Dis 56:1-25

2. Moran E, Masters S, Berendt AR, McLardy-Smith P, Byren I, Atkins BL (2007) Guiding empirical antibiotic therapy in orthopaedics: the microbiology of prosthetic joint infection managed by debridement, irrigation and prosthesis retention. J Infect 55:1-7

3. Sousa R, Pereira A, Massada M, da Silva MV, Lemos R, Costa e Castro J (2010) Empirical antibiotic therapy in prosthetic joint infections. Acta Orthop Belg 76:254-259

4. Sheehy SH, Atkins BA, Bejon P, Byren I, Wyllie D, Athanasou NA, Berendt AR, McNally MA (2010) The microbiology of chronic osteomyelitis: prevalence of resistance to common empirical anti-microbial regimens. J Infect 60:338-343

5. Teterycz D, Ferry T, Lew D, Stern R, Assal M, Hoffmeyer P, Bernard L, Uçkay I (2010) Outcome of orthopedic implant infections due to different staphylococci. Int J Infect Dis 14:913-918

6. Mortazavi SM, Vegari D, Ho A, Zmistowski B, Parvizi J (2011) Two-stage exchange arthroplasty for infected total knee arthroplasty: predictors of failure. Clin Orthop Relat Res 469:3049-3054

7. Vandecasteele SJ, Peetermans WE, Carbonez A, Van Eldere J (2004) Metabolic activity of Staphylococcus epidermidis is high during initial and low during late experimental foreign-body infection. J Bacteriol 186:2236-2239

8. Uçkay I, Harbarth S, Peter R, Lew D, Hoffmeyer P, Pittet D (2010) Preventing surgical site infections. Expert Rev Anti Infect Ther $8: 657-670$

9. Harbarth S, Samore MH, Lichtenberg D, Carmeli Y (2000) Prolonged antibiotic prophylaxis after cardiovascular surgery and 
its effect on surgical site infections and antimicrobial resistance. Circulation 101:2916-2921

10. Malekzadeh D, Osmon DR, Lahr BD, Hanssen AD, Berbari EF (2010) Prior use of antimicrobial therapy is a risk factor for culture-negative prosthetic joint infection. Clin Orthop Relat Res 468:2039-2045

11. Performance standards for antimicrobial susceptibility testing (2007) 17th informational supplement (standard M100-S17). Clinical and Laboratory Standards Institute, Wayne

12. Vittinghoff E, McCulloch CE (2007) Relaxing the rule of ten events per variable in logistic and Cox regression. Am J Epidemiol 165:710-718

13. Uçkay I, Lübbeke A, Harbarth S, Emonet S, Tovmirzaeva L, Agostinho A, Longtin Y, Peter R, Hoffmeyer P, Pittet D (2012) Low risk despite high endemicity of methicillin-resistant Staphylococcus aureus infections following elective total joint arthroplasty: a 12-year experience. Ann Med 44:360-368

14. Uçkay I, Harbarth S, Ferry T, Lübbeke A, Emonet S, Hoffmeyer P, Pittet D (2011) Meticillin resistance in orthopaedic coagulasenegative staphylococcal infections. J Hosp Infect 79:248-253

15. Cranny G, Elliott R, Weatherly H, Chambers D, Hawkins N, Myers L, Sculpher M, Eastwood A (2008) A systematic review and economic model of switching from non-glycopeptide to glycopeptide antibiotic prophylaxis for surgery. Health Technol Assess 12:iii-iv, xi-xii, 1-147

16. Berbari EF, Marculescu C, Sia I, Lahr BD, Hanssen AD, Steckelberg JM, Gullerud R, Osmon DR (2007) Culture-negative prosthetic joint infection. Clin Infect Dis 45:1113-1139

17. Zimmerli W, Trampuz A, Ochsner PE (2004) Prosthetic-joint infections. N Engl J Med 351:1645-1654 